\title{
Automatic Non-linear MRI-Ultrasound Registration for the Correction of Intra-operative Brain Deformations
}

\author{
Tal Arbel ${ }^{1}$, Xavier Morandi $^{1}$, Roch M. Comeau $^{2}$, and D. Louis Collins ${ }^{1}$ \\ 1 Montreal Neurological Institute, McGill University, Montréal, Québec, Canada \\ \{taly, morandi, louis\}@bic.mni.mcgill.ca, http://www.bic.mni.mcgill.ca \\ 2 Rogue Research Inc., 4398 St-Laurent, Suite 206, Montréal, Québec, Canada \\ roch@rogue-research.com, www.rogue-research.com
}

\begin{abstract}
Movements of brain tissue during neurosurgical procedures reduce the effectiveness of using pre-operative images for intra-operative surgical guidance. In this paper, we explore the use of acquiring intraoperative ultrasound (US) images for the quantification of and correction for non-linear brain deformations. We will present a multi-modal, automatic registration strategy that matches pre-operative images (e.g. MRI) to intra-operative ultrasound to correct for the non-linear brain deformations. The strategy involves using the predicted appearance of neuroanatomical structures in ultrasound images to build "pseudo ultrasound" images based on pre-operative segmented MRI. These images can then be registered to intra-operative US in a strategy based on crosscorrelation measurements generated from the ANIMAL 1 registration package. The feasibility of the theory is demonstrated through its application to clinical patient data acquired during 12 neurosurgical procedures. Qualitative examination of the results indicate that the system is able to correct for non-linear brain deformations.
\end{abstract}

Key words: Image-guided Neurosurgery, ultrasound, magnetic resonance, brain shift, multi-modal registration, non-linear registration

\section{Introduction}

The precise localization of anatomical structures and pathological features within the complex three-dimensional architecture of the brain is one of the major challenges of neurosurgery. Image-guided neurosurgical (IGNS) systems that embed neuronavigation are becoming widely used for intra-cranial procedures [2, 3]. There are numerous benefits reported from the increased usage of IGNS. These include (i) minimally invasive cranial openings, (ii) accurate localization of subcortical lesions, (iii) reduction in blood loss, (iv) reduction in surgical time, and (v) a decrease in the complication rate and thus on intensive care unit and hospital stays [4, 5]. However, the requirements for a precise, reliable and accurate surgical guidance system include: low system error and a high degree 
of correspondence between the pre-operative images and the surgical anatomy. Errors in IGNS caused by geometrical distortions in the pre-operative images, patient-to-image registration errors and errors in tracking the surgical instruments are well-defined for various systems in existence [3, 6]. However, another important source of error is the deformation of the brain tissue from the time of pre-operative image acquisition to the time of the surgical procedure and, more importantly, during the surgery. Brain deformation is a complex, spatiotemporal phenomenon with a wide variety of causes, including both chemical and physical factors [3, 4, 7]. IGNS systems based on pre-operative data alone cannot properly account for this type of deformation, which is estimated to range from $5 \mathrm{~mm}$ to $20 \mathrm{~mm}$ [4, 7].

In order to quantify and correct for spatial errors resulting from intraoperative brain deformations, registration strategies based on intra-operative MRI have been proposed and implemented 8, 9]. This approach has several strengths and weaknesses. Among the advantages are safer tumor resections, a better assessment of the resections, the quantification and visualization of brain deformations, and detection of intra-operative complications such as hemorrhage. Nevertheless, a number of technical and logistical problems still require solutions. These include restricted surgical access to the patient due to the confining layout of the MR system, the shortage of MR compatible auxiliary equipment and surgical instruments, and inconsistent image quality. Finally, the significant expense of the solution, both in terms of acquisition equipment and MRI-friendly surgical tools, leads to questions regarding the cost-effectiveness of the solution 9 .

Another recently proposed approach for the correction of deformations consists of using pre-operative MR images to generate a 3D patient-specific model of the brain resulting from simulated surgical procedures [10, 11]. Since the causes of brain deformations are complex, predicting the physical and chemical processes that can be encountered in each case would be difficult. Moreover, the presence of pathologies increases the complexity of the processes, particularly cases that lead to tissue removal and water displacement (e.g. cerebrospinal fluid, edema, etc).

Ultrasound (US) has a long established track record for intra-operative use in neurosurgical practice. Intra-operative US systems cost less than $10 \%$ of a typical MRI system, are portable (enabling them to be shared), have few special logistical requirements and are compatible with existing operating room equipment [12. Nevertheless, owing to limited image quality and limited experience in interpreting such images, the first period of enthusiasm in the 1980s was followed by disappointment. Since the mid-1990s, Trobaugh et al. [13], followed by other researchers [14, 15, 16, 12, 17] have developed the concept of correlating intra-operative US with pre-operative MRI using 2D and 3D images. Some techniques have been developed to match pre-operative MR images to intraoperative US to correct for linear deformations [19]. However, to the best of our 
knowledge, this paper presents the first report of an automatid 1 non-linear registration technique that successfully corrects for non-linear brain deformations. It should be noted that other groups have also developed non-linear correction methods, but they depend on manually identified landmarks to drive the warp [18. In our approach, we propose using a surgical guidance system that incorporates an automatic non-linear MRI-US registration method for the correction of intra-operative brain deformations. The feasibility of the theory has been demonstrated in a clinical context on a series of 12 patients.

\section{Methods}

The goal of this work is to update the patient's pre-operative MR images based on US images acquired intra-operatively, given the presence of non-linear brain deformations. Our strategy is to first acquire pre-operative patient MRI and store the result as a $3 \mathrm{D}$ volume. During the surgical procedure, a series of US images are acquired. The specific aims of this work are: (i) to compute an initial linear transformation that maps the position and orientation of the US images into the coordinate space defined by the pre-operative MR volume, (ii) to construct a full $3 \mathrm{D}$ composite US volume from the images acquired and (iii) to compute the nonlinear deformation field from the US volume to the corresponding MR volume in order to correct for non-linear brain deformations as well as errors in the linear registration stage. The resulting deformation field will be used to provide the surgeon with an update of the patient's MR images during the procedure. In this section, we will describe the various processing steps required to meet these goals.

\subsection{Linear Registration}

Pre-operative patient MRI are acquired and stored as a $3 \mathrm{D}$ volume in MINC format, a publicly available medical image file format developed at the Montreal Neurological Institute that was designed as a multi-modal, N-dimensional, crossplatform format 20. The file format is self-describing, portable and simplifies inter-process communication through transform files that allow one data set to be mapped to another.

In order to perform comparisons between the two data sets, US images acquired intra-operatively must then be mapped to the same space as the corresponding pre-operative MRI. Three rigid body or affine transformations are required to perform the mapping, as described in 16, by making use of a Polaris tracking system (Northern Digital Inc.). At the start of the surgical procedure, MR image space is mapped to the Polaris reference space by identifying homologous anatomical landmarks on the patient head using a tracked pointer, and on the MR images using a mouse, and employing a least squares (SVD) minimization fit. Normally, five to nine points are used to define the transformation.

\footnotetext{
${ }^{1}$ The registration strategy is fully automatic, however pathologies must be manually
} processed pre-operatively. 
During the surgical procedure, the tracker monitors the orientation and position of the US transducer through a tracked rigid body, or "US tracker", permanently mounted on the US transducer. US images are implicitly acquired in Polaris reference space as the US image-to-US tracker transformation is determined prior to surgery, using a calibration tool designed for this purpose, and the tracker itself provides the transformation from US tracker to the Polaris reference space. Thus, as US images are acquired during surgery, the resulting transformation can be used to extract a corresponding oblique slice from the MRI volume, permitting the simultaneous display and comparison of the pre-operative MRI and intra-operative US. The system stores the coordinates of the acquired US image in the appropriate coordinate space within the MINC format.

\subsection{D Composite US Volume}

Once the sequence of acquired US image 2 are stored in the appropriate coordinate space, a composite 3D US is created by superimposing and averaging the slices into a full 3D volume (originally zero-valued) in the same coordinate frame as the MR volume acquired earlier. The methodology chosen uses a nearest neighbour approach to place each intensity pixel into the nearest voxel in the volume image, in a strategy similar to that in [21]. Because the images are stored in MINC format, tools are available to average the slices in the areas where they intersect. A blurring operator is applied to the volume to remove the effects of acoustic artifacts such as speckle noise. An example of an US volume superimposed onto a patient's MRI can be seen in Figure 1

\subsection{Non-linear Registration}

In previous work, we have developed a versatile $3 \mathrm{D}$ volume registration and segmentation package, termed ANIMAL (Automatic Non-linear Image Matching and Anatomical Labeling) [22, 1]. In this project, ANIMAL is primarily used to compute the non-linear spatial transformation required to map intra-operative US to pre-operative MRI. The procedure for estimating this transformation is to compute dense field of $3 \mathrm{D}$ deformation vectors, mapping each voxel of one image volume to match those in a target image volume. The algorithm builds up a 3D non-linear deformation field in a piecewise linear fashion, recursively fitting local spherical neighborhoods. Each local neighborhood from one volume is translated to achieve an optimal match within the other volume using crosscorrelation similarity metrics to estimate the local transformation. The local neighborhoods are arranged on a 3D grid to fill the volume and each grid node moves within a range defined by the grid spacing. This procedure is applied in a hierarchical fashion, first using blurred data to estimate the largest and most global deformations, and then refining the transformation by using less blurred data and finer grid sampling to account for more local deformations. The process stops when reaching a $2 \mathrm{~mm}$ grid size.

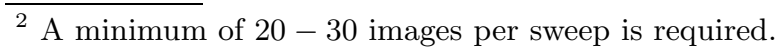


ANIMAL requires similar features in the source and target volumes in order to perform either cross-correlation or optical flow computations between the two data sets. Since US and MRI have very different characteristics, we have decided to generate pseudo US images - images whose appearance closely matches the predicted appearance of real US images that will be acquired during surgery - from data derived from the pre-operative MRI ${ }^{3}$. In this manner, the ANIMAL routine can be used to correlate the pseudo US to the real, intra-operative US images acquired. To compute the pseudo US volumes, the ANIMAL segmentation package is used to segment major brain structures from the MRI volume. The segmentation is then further refined to create a volume that includes only those structures that are clearly visible in US. The resulting volume of anatomical structures is then submitted to a radial gradient operator in order to generate gradient magnitude data that reflects the appearance (in terms of intensity values) of acoustic boundaries visible in the US image. In future work, other structures prevalent in the US image (such as the cerebral falx) will be added to the pseudo US images, and more realistic physical simulations will be developed. However, the current technique allows for a proof-of-principal to be demonstrated here. For the time being, pre-operative segmentation of pathologies is performed manually 4 . Figure 2 shows an example of each of the steps involved in creating a pseudo US image $\left(0.5 \mathrm{~mm}^{2}\right.$ pixel size $)$.

ANIMAL then estimates the non-linear spatial transformation required to match a pseudo US image volume to real US images acquired during surgery. This same transformation can be used to update the patient's MRI during surgery, thus permitting the neurosurgeon to make use of the pre-operative images during the intervention, even in the presence of a brain deformation (and errors in the linear registration from the patient to the pre-operative images).

\section{Clinical Applications}

The method was applied to 12 surgical cases, including those with brain tumors $(n=8)$ and selective amygdalo-hippocampectomies $(n=4)$. Pre-operative MRI were acquired using a Philips 1.5T Gyroscan (The Netherlands) machine. Intraoperative images were acquired using an Ultramark 9, Advanced Technologies Laboratories Inc. (Bothwell, WA) machine with an ATL P7-4 multi-frequency probe, and a Capsure ${ }^{T M}$ frame grabber on a Macintosh computer (Apple Computer, Cupertino, CA). Tracking was achieved with the use of a Polaris tracking system with a passive probe.

The feasibility of the approach is demonstrated through examination of the qualitative results from two clinical cases. Figure 4 shows the case of an amygdalo-hippocampectomy for intractable epilepsy. US images were acquired at two different stages of the operation: before and after the opening of the dura. The figure illustrates how the system is able to correct for brain deformations at these two stages of surgery. The extent of the correction can be seen in

\footnotetext{
${ }^{3}$ The current strategy does not take acoustic properties or physics into account.

${ }^{4}$ The automatic segmentation of pathologies is currently an open research topic.
} 
Figure 3 . Figure 5 illustrates the case of a tumor resection. Here, US images were acquired after the dura opening, when significant brain deformation (on the order of $8 \mathrm{~mm}$ ) had occurred. The strategy was able to correct for the deformations of both pathological and anatomical structures. In all cases, it took approximately 30 seconds of processing time to provide a corrected MRI volume on average.

\section{Discussion and Conclusions}

In the context of image-guided craniotomies for brain lesions, there are three important issues to address: (i) finding the lesions, (ii) avoiding the eloquent, functional brain tissue, and (iii) determining the borders between pathological and normal brain tissues. Over the past decade, advances in IGNS systems have permitted neurosurgeons to reach these objectives in a large number of situations 2]. However, because of the frequent occurrence of brain deformations during craniotomies, the accuracy of the neuronavigation system is often compromised. In this paper, we have demonstrated preliminary clinical results for the automatic correction of brain deformations during craniotomies by using a non-linear registration strategy that automatically matches pre-operative MRI to intra-operative US. The strategy is based on cross-correlation computations within ANIMAL that register pseudo US images, derived from segmented pre-operative MRI, to intra-operative US images. Current work involves building more sophisticated US simulations, based on the identification of a larger number of neuro-anatomical structures in clinical US data, and better physical modeling of the acoustic phenomenon.

One important advantage of the intra-operative system described in this paper is its relative speed. Pre-operative processing time for the creation of the pseudo US image, including the segmentation and gradient computations, is less than 10 minutes. The linear registration is a one-step process that lasts less than 5 minutes. Intra-operative computations include: performing the US acquisition ( $2-3$ minutes), computing the composite US volume (2 minutes) and finally, computing the deformations and updating the MRI takes 30 seconds. Optimization of the US composition should take that step down to several seconds.

The standard criticism associated with the use of US during neurosurgical procedures are its poor spatial and contrast resolutions. Our approach minimizes these concerns by providing the neurosurgeon with high resolution MR, warped to reflect the surgical reality, based on US images acquired intra-operatively. The technique has been applied to the images of 12 patients to demonstrate the feasibility and the reliability of the method. Further work is required to quantitatively validate the method, as well as to assess the added clinical value to the patient outcomes. Future work will also include a quantification of the registration error, and an interface to provide the neurosurgeon with the means to participate in the error control process.

\footnotetext{
${ }^{5}$ Figure references are not in sequence to ensure that all color figures appear on the same page.
} 


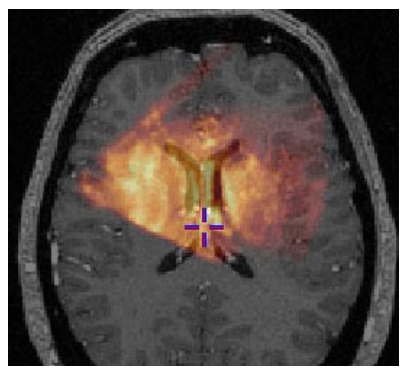

(a) Axial view

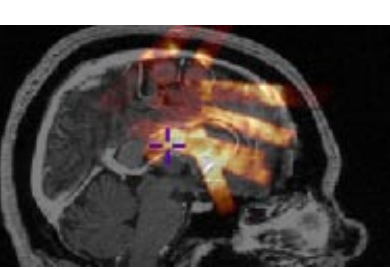

(b) Saggital view

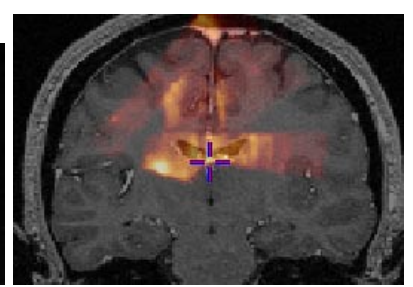

(C) Coronal view

Fig. 1. 3D Composite US Volume: MRI in grey with US overlayed in a hot-metal color scale.

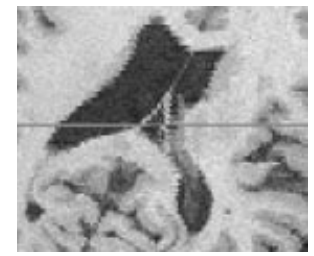

Original MRI

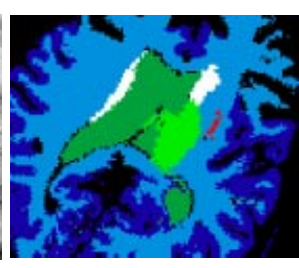

Segmented MRI

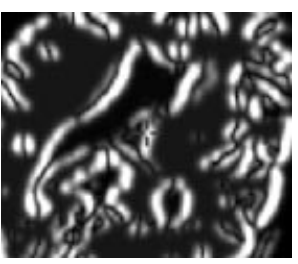

Gradient MRI

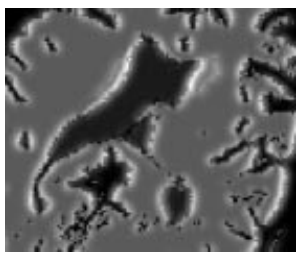

Pseudo US

Fig. 2. Generating Pseudo US. The original MRI is segmented using ANIMAL, a radial gradient is then applied, and these are merged to create a pseudo US image (see text for details).
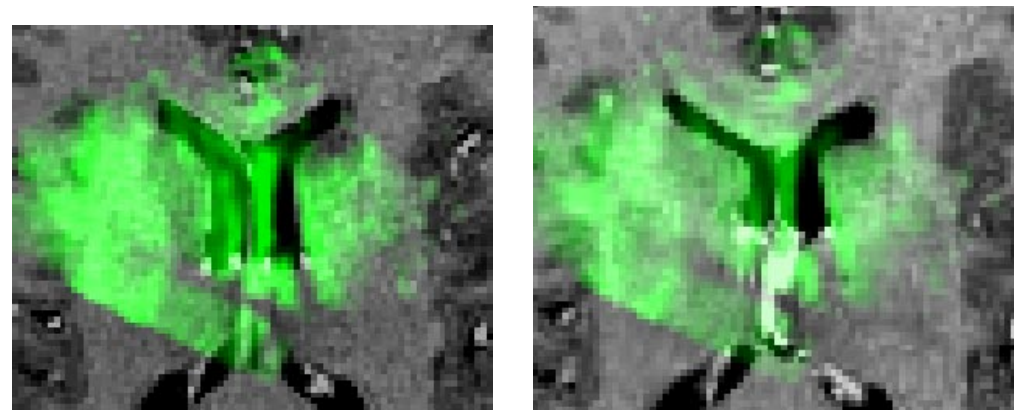

Fig. 3. Case from Figure 4illustrating US (in green) after dura opening over original MRI (left) and over corrected MRI (right). Notice the distinct collapse of the left lateral ventricle. 


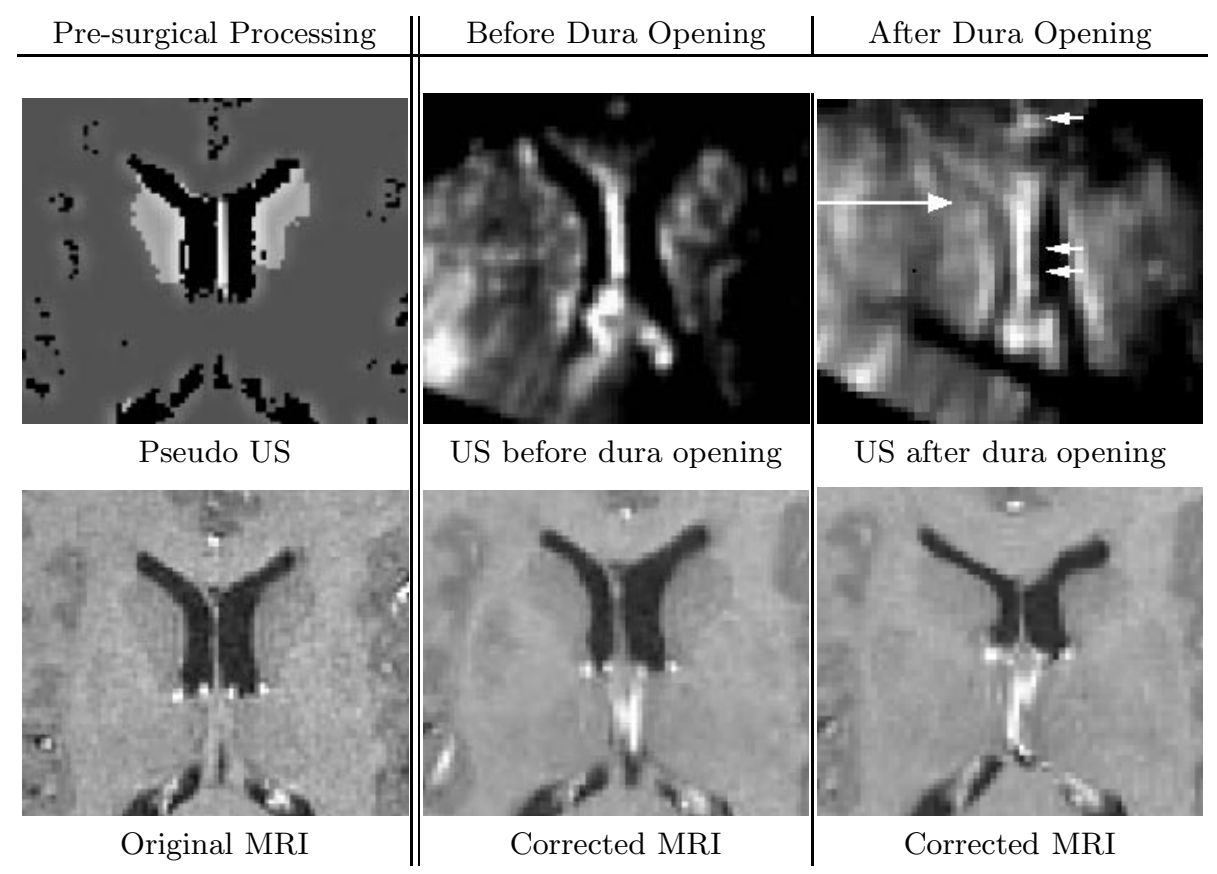

Fig. 4. Left selective amygdalo-hippocampectomy for intractable epilepsy: zoom of transverse images through the lateral ventricles. Patient was in the supine position with the head turned on the right side. A slight brain deformation is visible before the dura opening (column 2). A larger gravitational displacement (towards the right of the image) of the median structures is observed after dura opening (column 3). The deformation mainly involves the anterior horn of the left lateral ventricle (white arrow), whereas the falx (arrowhead) and septum pellucidum (double arrowhead) do not move. Correction of the deformation is demonstrated during these two surgical steps.

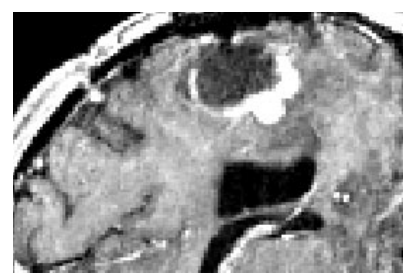

(1) Original MRI

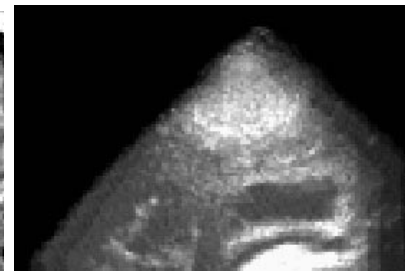

(2) Intra-operative US

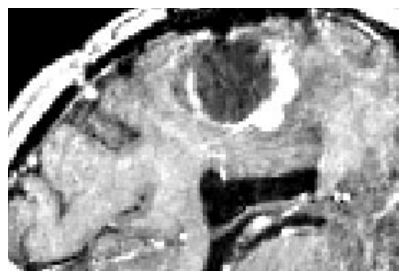

(3) Corrected MRI

Fig. 5. Case with right frontal recurrent malignant tumor. These near-transverse images show the tumor (top), ventricles (bottom), with the front of the head towards the right. After dura opening, the sinking of the entire tumor, as well as the deeplyseated median structures, are clearly visible as a displacement towards the bottom of the image. The MRI is corrected for deformations of both pathological and anatomical structures (e.g. The ventricle is displaced and slightly compressed. The tumor is displaced as well.) The posterior part of the septum drops, but the anterior part does not as the registration system confuses it with the choroid plexus. This will be fixed with proper representation of the septum and choroid plexus in the simulations. Note that the falx does not move. 


\section{References}

[1] D. L. Collins and A. C. Evans, "Animal: validation and applications of non-linear registration-based segmentation," International Journal and Pattern Recognition and Artificial Intelligence, vol. 11, pp. 1271-1294, Dec 1997.

[2] N. Dorward, "Neuronavigation - the surgeon's sextant," British Journal of Neurosurgery, vol. 11, pp. 101-103, 1997.

[3] J. Golfinos, B. Fitzpatrick, L. Smith and R. Spetzler, "Clinical use of a frameless stereotactic arm: result of 325 cases," Neurosurgery, vol. 83, pp. 197-205, 1995.

[4] D. Hill, C. Maurer, R. Maciunas et al., "Measurement of intraoperative brain surface deformation under a craniotomy," Neurosurgery, vol. 43, pp. 514-528, 1998.

[5] T. Paleologos, J. Wadley, N. Kitchen, and D. Thomas, "Clinical utility and cost-effectiveness of interactive image-guided craniotomy: clinical comparison between conventional and image-guided meningioma surgery," Neurosurgery, vol. 47, pp. 40-48, 2000.

[6] C. Maurer, G. Aboutanos, B. Dawant et al., "Effect of geometrical distortion correction in MR on image registration accuracy," Journal of Computer Assisted Tomography, vol. 20, pp. 666-679, 1996.

[7] D. Roberts, A. Hartov, F. Kennedy et al., "Intraoperative brain shift and deformation: a quantatative analysis of cortical displacement in 28 cases," Neurosurgery, vol. 43, pp. 749-760, 1998.

[8] P. Black, E. Alexander, C. Martin et al., "Craniotomy for tumor treatment in an intraoperative magnetic resonance unit," Neurosurgery, vol. 45, pp. 423-433, 1999.

[9] M. Bernstein, A. Al-Anazi, W. Kurcharczyk et al., "Brain tumor surgery with the Toronto open magnetic resonance imaging system: preliminary results for 36 patients and analysis of advantages, disadvantages, and future prospects," Neurosurgery, pp. 900-907, 2000.

[10] D. Roberts, M. Miga, A. Hartov et al., "Intraoperatively updated neuroimaging using brain modeling and sparse data," Neurosurgery, vol. 45, pp. 1199-1207, 1999.

[11] D. Roberts, M. Miga, A. Hartov et al., "Model-updated image guidance: Initial clinical experiences with gravity-induced brain deformation," IEEE Transactions on Medical Imaging, vol. 18, pp. 866-874, Oct 1999.

[12] R. Comeau, A. Sadikot, A. Fenster, and T. Peters, "Intraoperative ultrasound for guidance and tissue shift correction in image-guided neurosurgery," Medical Physics, vol. 27, pp. 787-800, 2000.

[13] J. Trobaugh, W. Richard, K. Smith, and R. Bucholz, "Frameless stereotactic ultrasonography: Methods and applications," Computerized Medical Imaging and Graphics, vol. 18, no. 4, pp. 235-246, 1994.

[14] R. Bucholz, C. Sturm, and J. Henderson, "Detection of brain shift with an image guided ultrasound device," Acta Neurochirurgica, vol. 138, p. 627, 1996.

[15] R. Bucholz, D. Yeh, J. Trobaugh et al., "The correction of stereotactic inaccuracy caused by brain shift using an intraoperative ultrasound device," CVRMedMRCAS, pp. 459-466, 1997.

[16] R. M. Comeau, A. Fenster, and T. Peters, "Intraoperative US in interactive imageguided neurosurgery," Radiographics, vol. 19, no. 4, pp. 1019-1027, 1998.

[17] D. Gobbi, R. Comeau, and T. Peters, "Ultrasound/MRI overlay with image warping for neurosurgery," in MICCAI 2000, (Pittsburgh, PA, USA), pp. 106-114, Oct. 2000 . 
[18] D. Gobbi, B. K. H. Lee, and T. M. Peters, "Correlation of preoperative MRI and intraoperative 3D ultrasound to measure brain tissue shift," in Medical Imaging 2001: Visualization, Display, and Image-Guided Procedures, Proceedings of SPIE 2001, (San Diego, CA, USA), Seong Ki Mun (ed.), Vol. 4319, pp. 264-271, Feb. 2001.

[19] A. Roche, X. Pennec, M. Rudolph et al., "Generalized correlation ratio for registration of 3D ultrasound with MR images," in MICCAI 2000, (Pittsburgh, PA, USA), pp. 567-577, Oct. 2000.

[20] P. Neelin, D. MacDonald, D. Collins, and A. Evans, "The MINC file format: from bytes to brains," NeuroImage, vol. 7, no. 4, p. 786, 1998.

[21] A. King, J. Blackall, G. Penney et al., "An estimation of intra-operative deformation for image-guided surgery using 3-D ultrasound," in MICCAI 2000 (Pittsburgh, PA, USA), pp. 588-597, Oct. 2000.

[22] D. L. Collins, P. Neelin, T. M. Peters, and A. C. Evans, "Automatic 3D intersubject registration of MR volumetric data in standardized Talairach space," Journal of Computer Assisted Tomography, vol. 18, pp. 192-205, 1994. 